\title{
Effective reduction of infarct volume by gap junction blockade in a rodent model of stroke
}

\author{
Ahmed Rawanduzy, M.D., Anker Hansen, M.D., Ph.D., Thomas W. Hansen, B.A., and Maiken \\ Nedergaard, M.D., Ph.D.
}

Departments of Neurosurgery and Cell Biology and Anatomy, New York Medical College; and Novo Nordisk, Målev, Denmark

Several lines of evidence indicate that the extent of ischemic injury is not defined immediately following arterial occlusion; rather that infarction expands over time. Episodes of spreading depression have been linked to this secondary increase in infarct volume. Tissue bordering the infarct fails to repolarize following spreading depression and is incorporated into the infarction. The result is that ischemic infarcts expand stepwise following each episode of spreading depression. Another line of evidence has demonstrated that gap junction blockers effectively inhibit spreading depression.

These observations suggest that the efflux of potentially harmful cytosolic messengers from ischemic cells into surrounding nonischemic cells might cause amplification of injury in focal stroke. It is therefore conceivable that minimizing gap junction permeability might reduce final infarct volume. To test this hypothesis, the authors pretreated rats with the gap junction blocker, octanol, before occluding the middle cerebral artery and compared the sizes of the ischemic lesions to those in rats that received vehicle dimethyl sulfoxide prior to arterial occlusion. Histopathological analysis was performed 24 hours later. The 12 octanol-treated animals showed a significantly decreased mean infarction volume $(80 \pm 16$ $\mathrm{mm}^{3}$ ) compared with the nine control rats $\left(148 \pm 9 \mathrm{~mm}^{3}\right)$. In a separate set of experiments, the frequency of experimentally induced waves of spreading depression was evaluated following octanol treatment. Octanol pretreatment resulted in complete inhibition in two of nine animals, transient inhibition in five of nine, and no inhibition in two of nine.

The results indicate that gap junction inhibitors, when not limited by toxicity, have significant therapeutic potential in the treatment of acute stroke.

Key Words * ischemia * middle cerebral artery occlusion * spreading depression * astrocyte * anoxic depolarization

Two intriguing observations have recently been made in stroke research: 1) the outer region of an ischemic infarct, the ischemic penumbra, is potentially salvageable tissue; and 2) waves of infarct-induced spreading depression invade the penumbra and their frequency and severity correlate with the ultimate extent of structural damage. $[8,16]$ If left untreated, an ischemic lesion will 
progressively expand and sequentially recruit surrounding areas in which there is compromised function and marginal blood flow into the infarct itself.[21] This process appears to be, at least in part, a result of the failure of tissue within the penumbra to repolarize following spontaneous waves of spreading depression.[5] Although these depolarizing waves are generated within ischemic regions, their invasion of neighboring tissue is widespread and can greatly enlarge stroke volume. $[9,14,17]$ A key step in understanding why an ischemic infarct expands is to establish why and how waves of spreading depression are generated within ischemic tissue. Recently we demonstrated that an intact gap junction network is required to propagate spreading depression.[15] In the present study we asked the question: Does administration of a gap junction blocker, octanol, decrease infarct volume following middle cerebral artery (MCA) occlusion in rats? We found that octanol effectively reduced infarct volume by $60 \%$ and partially inhibited spreading depression.

\section{MATERIALS AND METHODS}

\section{Animal Selection}

Twenty-one male Wistar rats, weighing between 330 and $360 \mathrm{~g}$, were used in this study. The animals were starved the night before surgery, but had free access to tap water.

\section{Presurgical Preparation}

The rats were anesthetized by an intraperitoneal injection of pentobarbital ( $50 \mathrm{mg} / \mathrm{kg}$ body weight). A polyethylene catheter was placed in the tail artery to record blood pressure. Supplemental pentobarbital doses of $15 \mathrm{mg} / \mathrm{kg}$ were administered hourly. Rectal temperature was kept close to $37^{\circ} \mathrm{C}$ by means of a thermostatically controlled heating lamp. Blood gas levels, blood $\mathrm{pH}$, blood glucose, and mean arterial blood pressure were monitored regularly throughout the operation. Experiments were completed only if these physiological variables remained within normal limits. The normal limits for mean arterial blood pressure were set at 90 to $130 \mathrm{~mm} \mathrm{Hg}, \mathrm{PCO}_{2}$ at 30 to $50 \mathrm{~mm} \mathrm{Hg}, \mathrm{PO}_{2}$ at 100 to $150 \mathrm{~mm} \mathrm{Hg}$, arterial blood glucose at 6 to $10 \mathrm{mM}$, and arterial blood $\mathrm{pH}$ at 7.25 to 7.45 . Octanol $(5.4 \mathrm{mM} / \mathrm{kg})$ dissolved in dimethyl sulfoxide was administered intraperitoneally 1 hour before surgery. Control rats received vehicle (dimethyl sulfoxide only).

\section{Surgical Procedure}

The animals underwent subtemporal craniotomy and exposure of the right MCA. With the aid of a microscope, the MCA was coagulated by micropolar cautery and cut just before the origin of the most lateral lenticulostriate arteries.[23] At the end of the experiment, the tail artery catheter was removed and the wounds were sutured. The animals were maintained under continuous temperature control until they recovered fully from anesthesia.

\section{Histopathological Examination}

Twenty four hours following occlusion of the MCA, the animals were reanesthetized with pentobarbital and decapitated. The brains were frozen in liquid nitrogen. Sections, $24 \mu \mathrm{m}$ thick, were cut and stained with hematoxylin and eosin. Ten coronal sections were selected at defined anatomical levels. Infarcts on these sections were visualized on an inverted microscope using a X 1.2 lens. Images of each section were collected with a SIT camera and analyzed using Image-1 commercially available software. Infarct volume was calculated as: infarct volume $\left(\mathrm{mm}^{3}\right)=\mathrm{A}\left(\mathrm{mm}^{2}\right) \mathrm{X} \mathrm{d}(\mathrm{mm})$, where A is the area of infarction on individual sections and $\mathrm{d}$ is the distance between the sections. 


\section{Microelectrodes and Detection of Direct Current Potential}

Two burr holes were made in the right parietal bone for insertion of the microelectrodes. Glass electrodes with a tip diameter of 2 to $4 \mu \mathrm{M}$ were made and used as previously described.[17] The animals were connected to ground via a glass tube filled with $1 \mathrm{M} \mathrm{KCl}$, which was solidified in agar and placed in the neck musculature. The electrodes were connected via $\mathrm{Ag}-\mathrm{AgCl}$ leads to a different electrometer (input impedance approximately $10^{14} \mathrm{Mohms}$ ). The direct current potential was recorded using commercially available software on a Power Macintosh Computer. Spreading depression was triggered by means of an electrode with its tip broken back to a diameter of approximately $10 \mu \mathrm{m}$, which was filled with $2 \mathrm{M} \mathrm{KCl}$ and connected to a manual microsyringe pump. This electrode was inserted $500 \mu \mathrm{m}$ into the neocortex and spreading depression was initiated by injection of $50 \mathrm{nl}$ of the $\mathrm{KCl}$ solution. Waves of spreading depression were reliably evoked in the control condition (79 times of 79 injections).

\section{Statistical Analysis}

All data are presented as the mean \pm standard deviation. Analysis of variance was used to compare the means across the groups, whereas Scheffé's F test was used to determine which groups significantly differed from one another.

\section{Sources of Supplies and Equipment}

Wistar rats were obtained from Taconic, Inc., Germantown, NY. The inverted microscope, model IX70, was manufactured by Olympus, Tokyo, Japan. The SIT camera, model Dage-60, and Image-1 software were obtained from Universal Imaging, Inc., Westchester, PA. The glass electrodes were purchased from Sutter Instruments, Novato, CA. MacLab software and the Power Macintosh computer, model 7100/80 AV, were obtained from Apple computers, Cupertino, CA.

\section{RESULTS}

\section{Neurological Behavior After Occlusion of the MCA}

The animals' systemic parameters were kept within normal physiological range and no intergroup differences were observed. Following occlusion of the MCA all animals exhibited variable degrees of left-sided paresis. By the time of decapitation ( 24 hours later) the rats had regained most of their motor function. 


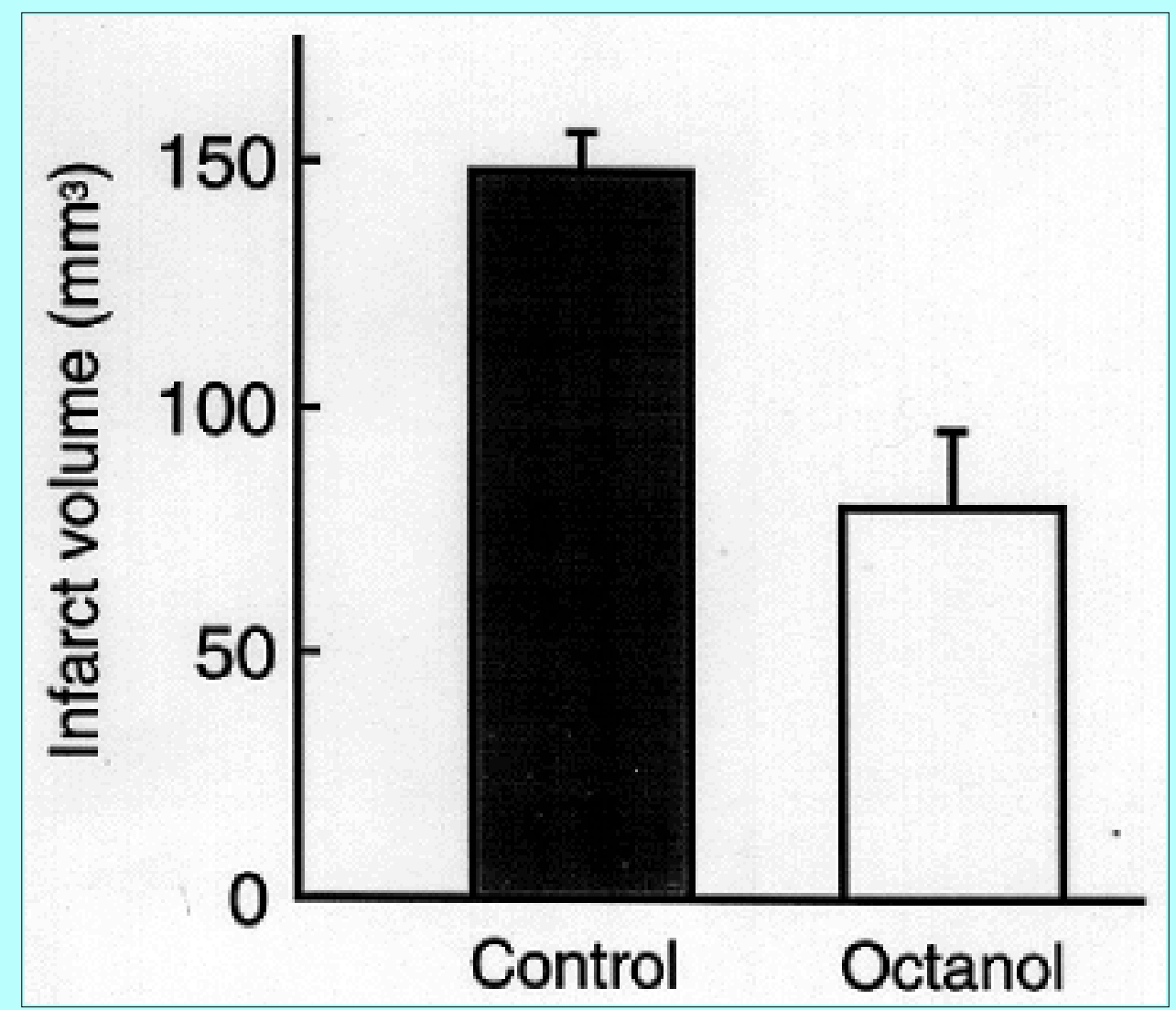

Fig. 1. Histogram depicting infarct volume (cubic millimeters) 24 hours after occlusion of the MCA in nine control and 12 octanol-treated rats. Octanol treatment reduced infarct volume by $60 \%(\mathrm{p}<0.01)$. Analysis of variance was used to compare the means across the two groups, and Scheffé's F test was used to determine significance level.

\section{Inhibition of Gap Junctions and Reduction of Infarct Volume}

The typical ischemic lesion produced in the octanol-treated group measured $80 \pm 16 \mathrm{~mm}^{3}$ compared with $148 \pm 9 \mathrm{~mm}^{3}$ in the control group (Fig. 1). The octanol-treated group exhibited significantly less damage than the nontreated group, suggesting that gap junction blockade effectively reduces infarct volume. Although the ischemic volume was dramatically reduced in the octanol-treated group, the general nature of the infarcts was similar in both groups. Infarcts were restricted to the lateral portion of the striatum and the lower and medial portions of the neocortex in both control and octanol-treated animals (Fig. 2). The striate infarcts showed little intraanimal variation; none extended into the medial striatum. In contrast, the size and extent of the cortical lesion was more variable with larger lesions consistently found in the control group. Cortical infarction was evident in nine of nine control rats, whereas only five of 12 octanol-treated rats developed significant cortical lesions. Thus, gap junction blockade significantly reduced the extent of cortical infarction, but had no detectable effect on the striate lesion. 


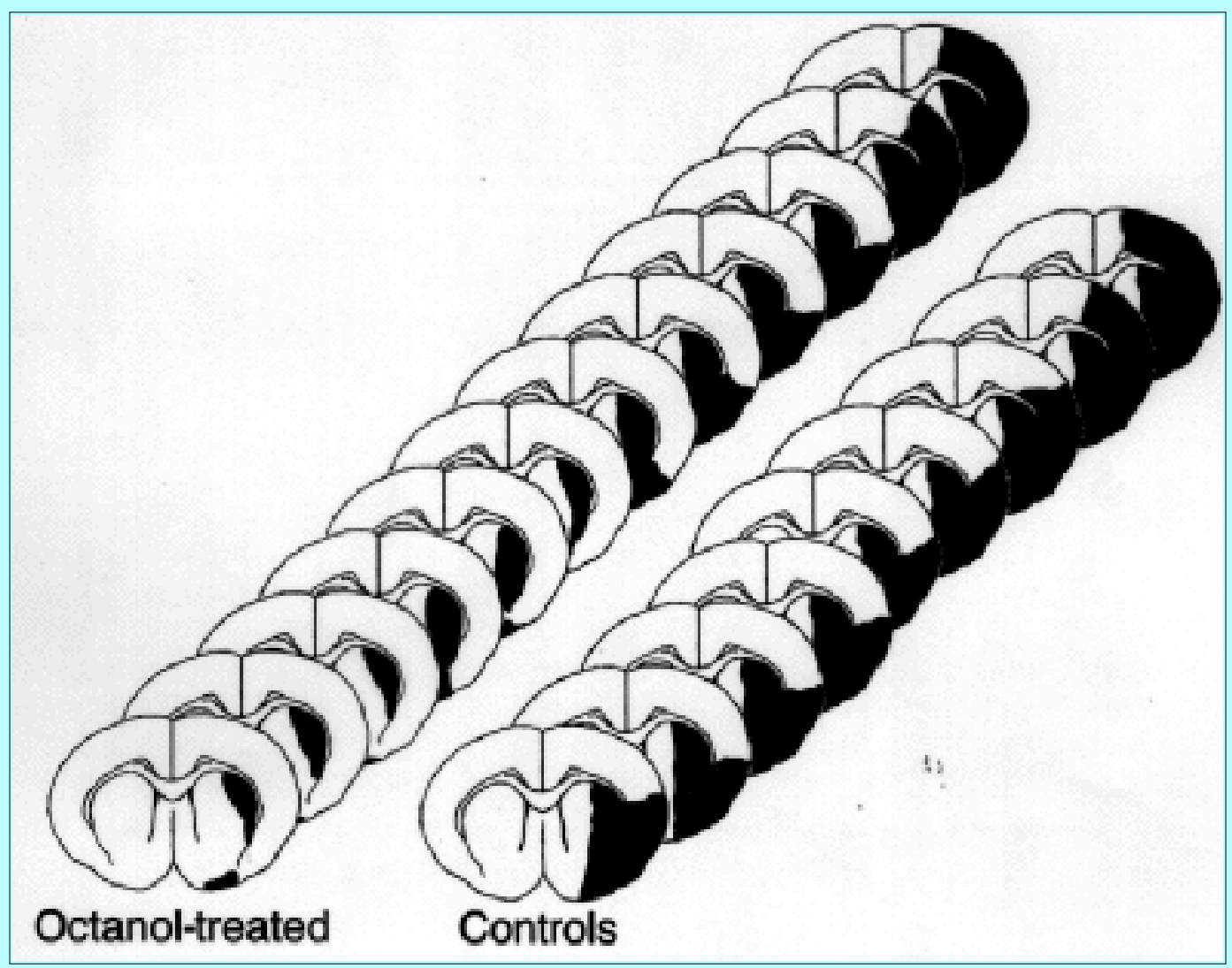

Fig. 2. Diagram illustrating the distribution of infarction 24 hours following occlusion of the MCA in individual animals. Infarction was restricted to the lateral parts of the striatum, with no animal showing expansion into the ventral striatum. In contrast, the size and extent of the cortical lesion varied from animal to animal and the cortical lesions were in general larger in the control group. Cortical infarction was evident in all nine rats in the control group, whereas only five of 12 rats developed significant cortical lesions in the octanol-treated group.

\section{Octanol Increased the Threshold for Generation of Spreading Depression}

Waves of spreading depression were evoked approximately every 15 minutes by injection of $\mathrm{KCl}$. After reliably evoking five to six waves in control conditions, octanol was administered intraperitoneally. Octanol completely blocked spreading depression in two of nine animals (Fig. 3). Inhibition occurred with a latency of 30 minutes following octanol treatment. Five of nine animals exhibited transient inhibition. During the period of inhibition, which lasted approximately 30 minutes, it was not possible to evoke waves of spreading depression even when the volume of the $\mathrm{KCl}$ injection was doubled; however, following this interval, waves of spreading depression could again be evoked. Two octanol-treated rats showed no sign of inhibition. Waves of spreading depression could be triggered reliably during a 2-hour observation period following administration of octanol. In all animals studied, octanol treatment did not effect the velocity or amplitude of direct current deflection when waves of spreading depression were evoked. Ischemic depolarization following cardiac injection of saturated $\mathrm{KCl}$ was not altered by octanol treatment. 


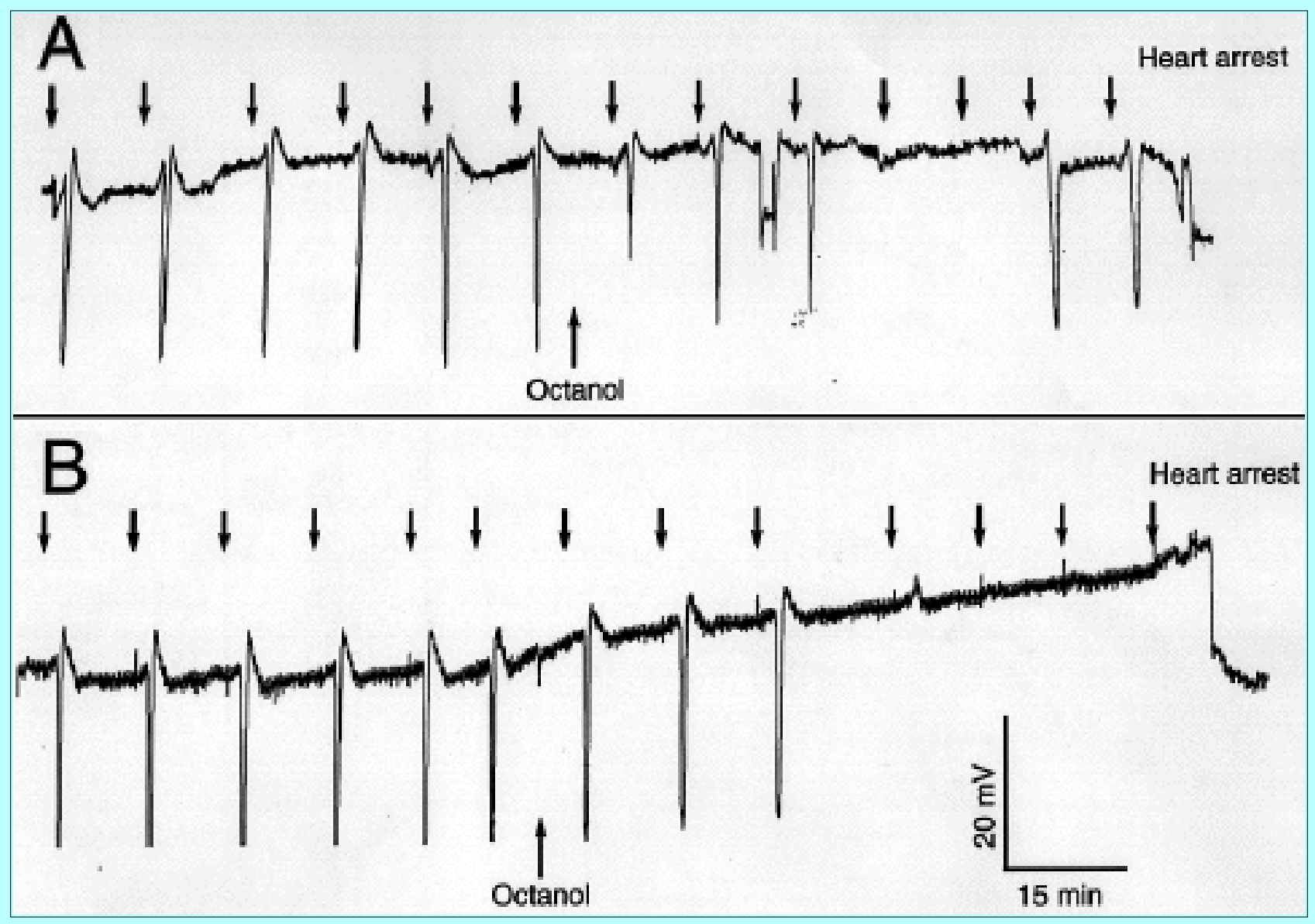

Fig. 3. Representative echocardiography demonstrating that spreading depression in the neocortex is inhibited by octanol. A: Repeated waves of spreading depression are evoked by injection of $\mathrm{KCl}$ (small arrows). Injection of $\mathrm{KCl}$ fails to evoke spreading depression 30 minutes following octanol treatment. Doubling the dose of KCL did not result in generation of spreading depression; however, spreading depression could be regenerated approximately 60 minutes following octanol administration. Normal appearing waves were evoked during the remaining observation period. B: Recording obtained in one representative animal in which generation of spreading depression was shown to be permanently inhibited 30 minutes following administration of octanol. Anoxic depolarization was not affected by octanol treatment in either of the animals.

\section{DISCUSSION}

This study demonstrates that gap junction blockade using octanol effectively reduces the size of ischemic lesions evoked by occlusion of the MCA in rats. In a separate set of experiments, it was also found that octanol treatment partially inhibits spreading depression. Together these observations support the notion that spreading depression contributes to expansion of ischemic damage. In addition, this study highlights the importance of astrocytes in the pathogenesis of ischemic infarction. Neurons only sparsely express gap junctions, whereas astrocytes are interconnected by an extensive network of gap junction channels.[3]

\section{Pathogenesis of Ischemic Infarction}

Occlusion of the MCA in rats produces a characteristic pattern of blood flow changes.[14,23] A core of very low or absent flow is bordered by tissue with partially reduced flow, known as the ischemic 
penumbra. Within minutes of the onset of ischemia, both neurons and astrocytes in the ischemic core are depleted of cellular energy metabolites and lose the ability to regulate their ion content. Both cell types are killed during the resultant ischemic infarction.[5,7,20] Ion homeostasis and cellular viability are not immediately compromised in the ischemic penumbra, but several lines of evidence have demonstrated that the ensuing infarct gradually expands into the ischemic penumbra. The expansion of the ischemic lesion is intimately linked with the occurrence of spontaneous waves of spreading depression.[9,14,21] Spreading depression is a slowly moving wave of tissue depolarization. Following occlusion of the MCA, waves of spreading depression are generated in the ischemic tissue. Once evoked, these waves migrate to include most of the ipsilateral hemisphere.[17] Tissue in the immediate vicinity of the evolving infarct does not repolarize after invasion of spreading depression, but remains depolarized and is eventually incorporated into the infarct.[8] The result is that the infarction grows stepwise into the ischemic penumbra following each episode of spreading depression.[12]

\section{What Is Spreading Depression?}

Spreading depression, classically described as the spreading depression of Leao, is a generalized response of vertebrate gray matter to a variety of noxious influences.[7] It constitutes a slowly moving wave of tissue depolarization in the intact brain. Spreading depression is experimentally evoked by applying $\mathrm{KCl}$ or the excitatory neurotransmitter, glutamate, to exposed cortical tissue or by electrical stimulation. Spreading depression is characterized by a reversible cessation of neuronal activity that propagates slowly (20-80 $\mu \mathrm{m} / \mathrm{second})$ and is accompanied by a loss of membrane potential and transmembrane ionic gradients.[18] In the neocortex, propagation of spreading depression can be tracked by inserting ion or potential-sensitive electrodes some distance from the focus of initiation. Both neurons and astrocytes participate in spreading depression. The slow propagation of these waves suggests that spreading depression is mediated by the diffusion of soluble factors.[6] Indeed, the $N$-methyl-D-asparatate receptor antagonists inhibit propagation of spreading depression, suggesting that glutamate is the diffusable messenger.[12] However, it was recently demonstrated that gap junction blockers, independent of $N$-methyl-D-asparatate receptor blockage, effectively inhibit propagation of spreading depression. This suggests that a gap junction-permeable cytosolic messenger might participate in the propagation of spreading depression.[15] In the brain, only astrocytes are interconnected by an extensive network of gap junctions, whereas other cell types including neurons are only sparely coupled.[3] Thus, it is not surprising that studies of cultured astrocytes have already lead to the description of a phenomenon that shares many characteristics with spreading depression.

\section{Is Spreading Depression an in Vivo Expression of Astrocytic Calcium Waves?}

Astrocytes are not electrically excitable and have traditionally been regarded as passive support cells. Their presumed function has been restricted to interstitial homeostasis, maintenance of the blood-brain barrier, and neurotrophin production.[22] This view of astrocytic function changed in 1990, when it was demonstrated that glutamate can trigger calcium oscillations among cultured glia, which often resulted in propagating intercellular calcium waves.[2] Astrocytic calcium waves propagate with a velocity of 10 to $20 \mu \mathrm{m} / \mathrm{second}$, whereas spreading depression propagates at 10 to $70 \mu \mathrm{m} / \mathrm{second}$. An intact gap junction network appears to be required for cel-to-cell calcium signaling, because agents that lower gap junction permeability, such as octanol and halothane, are effective blockers of calcium wave formation.[4] Further studies of this newly described intracellular signaling mechanism revealed that astrocytic calcium waves can modulate neuronal cytosolic calcium with large spikelike increases in neuronal calcium levels triggered by astrocyte signaling.[13] Thus, a signaling loop exists between cultured neurons and 
astrocytes: astrocytes can modulate the calcium level and thereby the firing pattern of neurons in their vicinity. In turn, neurons can trigger astrocytic calcium waves by releasing glutamate. Accordingly, gap junction blockers are effective inhibitors of astrocytic calcium signaling to cocultured neurons, but glutamate receptor antagonists have been similarly reported to dampen, although not eliminate, this signaling pathway.[19] Thus, several mechanisms might independently or synergistically act to mediate signaling between astrocytes and neurons.[22]

Spreading depression and astrocytic calcium waves share several characteristics such as velocity of propagation and triggering mechanism. Most notably, both spreading depression and astrocytic calcium waves can be triggered by glutamate, involve participation of both astrocytes and neurons, result in deregulation of calcium homeostasis, and require functional gap junctions.[16] Indeed, direct experimental evidence now supports the notion that astrocytic calcium waves constitute the leading edge of a propagating, spreading depression wave. It has been demonstrated that astrocytic calcium increments precede the depolarizing wave of spreading depression by several seconds in acutely prepared hippocampal slices.[11]

\section{How Might Astrocytic Gap Junctions Participate in Expansion of Ischemic Infarcts?}

Gap junctions are unique channels that connect a cell directly with the interior of other cells.[4] In the brain, gap junctions might serve as an important pathway for astrocytic calcium signaling.[25] In this study, we have demonstrated that administration of a gap junction blocker, octanol, effectively reduced infarct volume. Thus, a key step in understanding why an ischemic infarct gradually expands may be to establish how gap junction channels permit dying cells in the ischemic focus to communicate with each other, and in particular, with recoverable (viable) cells in the ischemic penumbra. Whether gap junction-mediated astrocytic signaling in vitro is an equivalent of spreading depression and thus contributes directly to expansion of ischemic infarction remains to be determined. Our study demonstrating that inhibition of astrocytic gap junctions reduces the extent of ischemic injury provides a novel perspective for dissection of the pathophysiology of acute stroke.

\section{References}

1. Astrup J, Siesjo B, Symon L: Thresholds in cerebral ischemia--the ischemic penumbra. Stroke 12:723-725, 1981

2. Cornell-Bell AH, Finkbeiner SM, Cooper MS, et al: Glutamate induces calcium waves in cultured astrocytes: long range glial signaling. Science 247:470-473, 1990

3. Dermietzel R, Spray DC: Gap juntions in the brain: where, what type, how many and why? Trends Neurosci 16:186-192, 1993

4. Finkbeiner S: Calcium waves in astrocytes--filling in the gaps. Neuron 8:1101-1108, 1992

5. Ginsberg M: Neuroprotection in brain ischemia: an update. The Neuroscientist 1:95-103, 1995

6. Grafstein B: Mechanism of spreading cortical depression. J Neurophysiol 19:154-171, 1956

7. Hansen AJ: Effect of anoxia on ion distribution in the brain. Physiol Rev 65:101-148, 1985

8. Hossmann KA: Viability thresholds and the penumbra of focal ischemia. Ann Neurol 36:557-565, 
9. Iijima T, Mies G, Hossmann KA: Repeated negative DC deflections in rat cortex following middle cerebral artery occlusion are abolished by MK-801: effect on volume of ischemic injury. J Cereb Blood Flow Metab 12:727-733, 1992

10. Lauritzen M, Hansen AJ: The effect of glutamate receptor blockade on anoxic depolarization and cortical spreading depression. J Cereb Blood Flow Metab 12:223-229, 1992

11. MacVicar B: New insights about glia in neurological disease. Winter Conference on Brain Research, 1996

12. Mies G, Iijima T, Hossmann KA: Correlation between peri-infarct DC shifts and ischaemic neuronal damage in rat. Neuroreport 4:709-711, 1993

13. Nedergaard M: Direct signaling from astrocytes to neurons in cultures of mammalian brain cells. Science 263:1768-1771, 1994

14. Nedergaard M, Astrup J: Infarct rim: effects of hyperglycemia on direct current potential and [14C]2-deoxyglucose phosphorylation. J Cereb Blood Flow Metab 6:607-615, 1986

15. Nedergaard M, Cooper AJ, Goldman SA: Gap junctions are required for the propagation of spreading depression. J Neurobiol 28:433-444, 1995

16. Nedergaard M, Goldman S: Spreading depression-a gap junction mediated event?, in Spray DC, Dermietzel R (eds): Gap Junctions in the Nervous System. 1996

17. Nedergaard M, Hansen AJ: Characterization of cortical depolarizations evoked in focal cerebral ischemia. J Cereb Blood Flow Metab 13:568-574, 1993

18. Nicholson C, Kraig RP: The behavior of extracellular ions during spreading depression, in Zeuthen T (ed): The Application of Ion-Selective Microelectrodes. Amsterdam: Elsevier, 1981

19. Parpura V, Basarsky TA, Liu F: Glutamate-mediated astrocyte-neuron signalling. Nature 369:744-747, 1994

20. Siesjo B: Pathophysiology and treatment of focal cerebral ischemia. I. Pathophysiology. J

Neurosurg 77:169-184, 1992

21. Siesjo BK, Zhao Q, Pahlmark K, et al: Glutamate, calcium, and free radicals as mediators of ischemic brain damage. Ann Thorac Surg 59:1316-1320, 1995

22. Smith SJ: Neural signalling. Neuromodulatory astrocytes. Curr Biol 4:807-810, 1994

23. Tamura A, Graham DI, McCulloch J, et al: Focal cerebral ischaemia in the rat: 1. Description of technique and early neuropathological consequences following middle cerebral artery occlusion. J Cereb Blood Flow Metab 1:53-60, 1981

Manuscript received March 10, 1997.

Accepted in final form April 19, 1997. 
This study was supported by National Institute of Neurological Disorders and Stroke and National Institutes of Health Grants RO130007 and RO135011. Dr. Nedergaard is an Established Investigator sponsored by The American Heart Association.

Address reprint requests to: Maiken Nedergaard, M.D., Department of Cell Biology and Anatomy, New York Medical College, Valhalla, New York 10595.

$\underline{\text { Click here to view Commentary on this article. }}$ 